OPEN ACCESS

Edited by:

Rashika El Ridi,

Cairo University, Egypt

Reviewed by:

Wai San Cheang,

University of Macau, China

Rasha Hanafi,

German University in Cairo, Egypt

*Correspondence:

Shuli Li

lishli@fmmu.edu.cn

Chunying $\mathrm{LI}$

lichying@fmmu.edu.cn

${ }^{\dagger}$ These authors have contributed equally to this work and share first authorship

${ }^{*}$ These authors share senior authorship

Specialty section: This article was submitted to Autoimmune and Autoinflammatory Disorders, a section of the journal

Frontiers in Immunology

Received: 19 December 2021 Accepted: 24 January 2022

Published: 10 February 2022

Citation:

Ye Z, Chen J, Du P, Ni Q, Li B, Zhang Z, Wang Q, Cui T, Yi X, Li C and Li S (2022) Metabolomics Signature and Potential Application of Serum Polyunsaturated Fatty Acids Metabolism in Patients With Vitiligo.

Front. Immunol. 13:839167. doi: 10.3389/fimmu.2022.839167

\section{Metabolomics Signature and Potential Application of Serum Polyunsaturated Fatty Acids Metabolism in Patients With Vitiligo}

\author{
Zhubiao $\mathrm{Ye}^{1 \dagger}$, Jianru Chen ${ }^{1 \dagger}$, Pengran $\mathrm{Du}^{1 \dagger}$, Qingrong $\mathrm{Ni}^{2}$, Baizhang $\mathrm{Li}^{1}$, Zhe Zhang ${ }^{1}$, \\ Qi Wang ${ }^{1}$, Tingting Cui ${ }^{1}$, Xiuli $\mathrm{Yi}^{1}$, Chunying $\mathrm{Li}^{1{ }^{1 \neq}}$ and Shuli $\mathrm{Li}^{1 \not \neq}$ \\ ${ }^{1}$ Department of Dermatology, Xijing Hospital, Fourth Military Medical University, Xi'an, China, ${ }^{2}$ Department of Dermatology,
The Medical Center of Air Force of People's Liberation Army, Forth Military Medical University, Beijing, China
}

Vitiligo is a depigmented skin disorder caused by a variety of factors, including autoimmune, metabolic disturbance or their combined effect, etc. Non-targeted metabolomic analyses have denoted that dysregulated fatty acids metabolic pathways are involved in the pathogenesis of vitiligo. However, the exact category of fatty acids that participate in vitiligo development and how they functionally affect $\mathrm{CD}^{+} \mathrm{T}$ cells remain undefined. We aimed to determine the difference in specific fatty acids among vitiligo patients and healthy individuals and to investigate their association with clinical features in patients with vitiligo. Serum levels of fatty acids in 48 vitiligo patients and 28 healthy individuals were quantified by performing ultra-performance liquid chromatographytandem mass spectrometry. Univariate and multivariate analyses were carried out to evaluate the significance of differences. Moreover, flow cytometry was used to explore the effect of indicated fatty acids on the function of $\mathrm{CD} 8^{+} \mathrm{T}$ cells derived from patients with vitiligo. We demonstrated that serological level of alpha-linolenic acid (ALA) was markedly upregulated, while that of arachidonic acid (ARA), arachidic acid (AA) and behenic acid were significantly downregulated in patients with vitiligo. Moreover, ALA levels were positively associated with vitiligo area scoring index (VASI) and ARA was a probable biomarker for vitiligo. We also revealed that supplementation with ARA or nordihydroguaiaretic acid (NDGA) could suppress the function of $C D 8^{+} T$ cells. Our results showed that vitiligo serum has disorder-specific phenotype profiles of fatty acids described by dysregulated metabolism of polyunsaturated fatty acids. Supplementation with ARA or NDGA might promote vitiligo treatment. These findings provide novel insights into vitiligo pathogenesis that might add to therapeutic options.

Keywords: metabolomics, arachidonic acid, alpha-linolenic acid, arachidonic acid metabolic pathway, serum, vitiligo 


\section{INTRODUCTION}

Vitiligo is an autoimmune skin disorder that presents as progressive depigmentation of skin due to the destruction of epidermal melanocytes caused by abnormal activation of $\mathrm{CD} 8^{+} \mathrm{T}$ cells (1). Multiple factors like metabolic abnormality, oxidative stress and phenolic compounds exposure were related to the pathogenesis of vitiligo (2-5). Growing evidence suggests that fatty acid metabolism is closely associated with autoimmune diseases, such as psoriasis (6), systemic lupus erythematosus (SLE) (7) and rheumatoid arthritis (RA) (8). Besides, it is reported that the incidence of metabolic syndromes in patients with vitiligo is significantly higher than that in healthy controls (9). However, the in-depth knowledge of vitiligo, in particular on the basis of metabolic dysregulation, remains unclear and need further exploration.

Metabolomics is an emerging field that be used to monitor the alternations in all low molecular metabolites produced by cellular processes, which delineates an overall metabolic profiles have widely applicable to investigate clinical features of various diseases. Recently, the metabolome based on miscellaneous samples, including serum, plasma, blister liquid and urine so on, have revealed that metabolic disturbance of amino acids and lipid mediators are involved in physiological and pathological changes in vitiligo patients (10-13). However, almost all previous studies on the basis of non-targeted metabolomics techniques, which the accuracy of quantitative methods are necessary to promote further. What's more, most of the research only focuses on the alternation of metabolites and dysregulated metabolic pathway, their specific role in the development and treatment various disorders still needed to further investigation. Therefore, targeted metabolomics method and confirmation experiments are of great significance to improve the accuracy of altered metabolites and to promote the management of vitiligo patients.

Several previous studies reported that supplementation with EPA and DHA contributes to the recovery of autoimmune diseases like RA and SLE (14-16). Another research disclosed that supplementation with ARA or aspirin, COX-2 inhibitor, also prevents healthy individuals from diabetes mellitus (17). What's more, growing evidence supports that omega- 3 polyunsaturated fatty acids are conducive to alleviate $\mathrm{CD}^{+} \mathrm{T}$ cell-mediated inflammatory response in various disorders $(18,19)$. Our previous non-targeted metabolomics study also found that levels of fatty acids manifested the most alterations among that of various metabolites. More importantly, the enriched Kyoto encyclopedia of gene and genomes (KEGG) pathway analysis revealed that several fatty acid metabolic pathways were significantly associated with vitiligo development (20). However, which specific fatty acid plays a key role and how it works in vitiligo remains ill-defined.

In our study, we conducted a targeted metabolomics assay to assess serum FAs concentration and demonstrated the major differentially expressed fatty acids in vitiligo, and further evaluated their correlation with clinical features. Additionally, we investigated the effect of ARA or its metabolic pathway on the activation and effect function of $\mathrm{CD} 8^{+} \mathrm{T}$ cells.

\section{MATERIALS AND METHODS}

\section{Study Subjects}

Serum for fatty acids profiles was derived from a continuous of sample of 76 participants: 48 vitiligo patients and 28 age- and gender-matched healthy controls. The diagnosis of vitiligo was ascertained and VASI scores assessed by dermatologists. Corresponding healthy volunteers were recruited from physical examination center of Xi Jing hospital to undertake the same testing as vitiligo patients. All study participants with active autoimmune diseases, malignancies, diabetes mellitus, pregnant and breastfeeding women were excluded from this study. Besides, study subjects who took glucocorticoids, antibiotics and other drugs or diet habit that might affected fatty acid metabolism in the past three months were ruled out as well. Epidemiological data including gender, age, disease duration, BMI, disease activity, clinical presentations, treatments, was face-to-face assessed and recorded by dermatologists. The VASI scores were evaluated by two senior dermatologists and calculated by average. Laboratory measurements including TC, TG, HDL-C and LDL-C were measured or collected from electronic patient record in hospital.

\section{Chemicals and Reagents Used for Metabolomics Analysis}

Detailed information of chemicals and reagents was described in the Supplementary Data.

\section{Sample Preparation and Fatty Acid Extraction}

Serum samples were obtained by centrifugation of fresh blood samples for $5 \mathrm{~min}$ at $2500 \mathrm{rpm}$ at $4^{\circ} \mathrm{C}$ and immediately quenched by liquid nitrogen and stored at $-80^{\circ} \mathrm{C}$ until further analyses. Prior to the assay, serum samples were left at $-20^{\circ} \mathrm{C}$ for 30 minutes and then thawed on ice-bath to diminish sample degradation. All fatty acid standards were prepared to storage solution at $5.0 \mathrm{mg} / \mathrm{mL}$. An appropriate amount of individual stock solution was mixed to obtain working standard solutions, which were made to produce the calibration curves. The fatty acid extraction method followed by a previously described report $(21,22)$. Briefly, $20 \mu \mathrm{l}$ of serum or each standard solution were mixed with $120 \mu \mathrm{l}$ cold methanol with internal standard solution were added to a $96-$ well plate. The mixed samples were centrifuged at $4000 \times \mathrm{g}$ at $4^{\circ} \mathrm{C}$ for $30 \mathrm{~min}$. An aliquot of $30 \mu \mathrm{l}$ the supernatant was transferred to a new 96-well plate. Subsequently, $20 \mu \mathrm{l}$ of derivative reagent, 3-nitrophenylhydrazine (3-NPH) was added to each well, the plate was locked and the derivatization was performed at $30^{\circ} \mathrm{C}$ for $60 \mathrm{~min}$. After derivatization, $400 \mu \mathrm{l}$ of ice-cold $50 \%$ methanol solution was added and stored at $-20^{\circ} \mathrm{C}$ for 20 minutes and followed by $4000 \times$ g centrifugation at $4^{\circ} \mathrm{C}$ for 30 minutes. Finally, the supernatant was used for LC-MS analysis.

\section{Analysis of Metabolites by UPLC-MS/MS}

Metabolomics profiling was performed using a UPLC-MS/MS platform (Acquity UPLC-Xevo TQ-S; Waters). The samples were randomized and analyzed using an Acquity UPLC BEH C18 1.7 $\mu \mathrm{M}$ VanGuard pre-column $(2.1 \times 5 \mathrm{~mm})$ and analytical column $(2.1 \times 100 \mathrm{~mm})$. The column temperature and sample manager 
temperature were maintained at $40^{\circ} \mathrm{C}$ and $10^{\circ} \mathrm{C}$, respectively. Water with $0.1 \%$ formic acid (solvent $\mathrm{A}$ ) and acetonitrile/IPA (70:30) (solvent B) were used as mobile phases. The flow rate and injection volume were $400 \mu \mathrm{l} / \mathrm{min}$ and $5 \mu \mathrm{l}$, respectively. The gradient condition was scheduled as follows: $0-1 \mathrm{~min}(50 \% \mathrm{~B}), 1$ $3.5 \mathrm{~min}(50-78 \% \mathrm{~B}), 3.5-12.2 \mathrm{~min}(78-100 \% \mathrm{~B}), 12.2-14.2 \mathrm{~min}$ (100\% B), $14.2-14.5 \min (100-50 \%$ B), $14.5-16 \min (50 \%$ B). The mass spectrometer was operated in the negative mode with a 2.0 $\mathrm{kV}$ capillary voltage. The source and desolvation temperatures were $150^{\circ} \mathrm{C}$ and $550^{\circ} \mathrm{C}$, respectively. And the desolvation gas flow was $1200 \mathrm{~L} / \mathrm{hr}$.

All samples were analyzed at beginning and end of each batch run and data for each ionization technique were gained in negative ion mode. The quality control (QC) samples were prepared by mixing equal volume of each serum sample (48 vitiligo samples, 28 healthy control samples) and then injected at regular intervals (after every 14 test samples for LC-MS) throughout the analytical run. Reagent blank samples consist of high purity solvents were randomly inserted among the real sample queue to serve as a useful alert to systematic contamination, as well as wash the column and remove cumulative matrix effects throughout the study.

\section{Analytical Validation}

The method validation was performed by the following ways: linearity and quantification limits, reproducibility and recovery of result. The detailed information are presented in Supplementary Data and Supplementary Table S1.

\section{Cell Separation and Culture}

Peripheral blood samples were collected by EDTA vacutainer tubes. Peripheral Blood Mononuclear Cells (PBMCs) were separated from the fresh blood samples of vitiligo patients by density-gradient centrifugation with lymphocyte separation medium. For CCK-8 assay, the $\mathrm{CD}^{+} \mathrm{T}$ cells were positively isolated from PBMCs with a magnetic-activated cell sorting $\mathrm{CD}^{+} \mathrm{T}$ Cell Isolation Kit (Miltenyi Biotec) according to the manufacturer's instructions. Cells were cultured in RPMI 1640 (Gibco) supplemented with 10\% fetal bovine serum (Gibco), and $1 \%$ penicillin-streptomycin solution.

\section{CCK-8 Assay}

The effect of ARA on the survival of human naïve $\mathrm{CD} 8^{+} \mathrm{T}$ cells were measured using the CCK- 8 assay based on the manufacturer's instruction. Briefly, the isolated $\mathrm{CD}^{+} \mathrm{T}$ cells were planked with $\left(10^{6}\right.$ cells $\left.\mathrm{mL}^{-1}\right)$ at 96 -well U-plate and incubated in the presence of different concentrations $(0,5,10,20,50,100 \mu \mathrm{M})$ of ARA for $48 \mathrm{~h}$. The viability of $\mathrm{CD}^{+}$T cells was detected by the CCK- 8 assay and the Optical Density was measured at $450 \mathrm{~nm}$ with a microplate reader.

\section{Flow Cytometry Analysis}

The detailed procedures of flow cytometry analysis are provided in Supplementary Data.

\section{Data Processing and Statistical Analysis}

In LC-MS/MS-based metabolomics analysis, the targeted raw data files were processed through using the MassLynx software (v4.1, Waters, Milford, MA, USA) to perform peak integration, calibration, and quantitation for each metabolite. The powerful package R studio and MetaboAnalyst 5.0 (a web-based software tool for metabolomic data analysis, https://www.metaboanalyst.ca/) were used for statistical analyses. To reduce the differences on the concentration of fatty acid between samples, auto-scaling was performed to make sure each variable comparable to each other. For multivariate statistical analysis, PLS-DA was used to visualized the difference between global metabolic profiles among the given groups that provides more valuable information, and RF algorithm was applied to enhance the quality of multivariate analyses and to avoid the risk of overfitting. For univariate analysis, discrete variables were summarized in percentage and compared between different groups using Chi-Square test or Fisher's exact test. Shapiro-Wilk test was applied to evaluate the distributions of continuous variables. Student's $t$-test or Mann-Whitney $U$ test were implemented to determine the statistical significance of each fatty acid, according to the distribution type of data. Pearson correlation analysis was carried out for clarifying the association between fatty acid and clinical characteristics.

ROC curves were performed to assess the diagnostic performance of fatty acids by MetaboAnalyst 5.0 and Graph Prism 8.0. Supporting vector machine and 10-fold crossvalidation was established to avoid the problem of potential overfitting in producing combined predicted ROC curves. Binary logistic regression was used to evaluate the risk factor of vitiligo.

For determining the potentially altered metabolic pathways in vitiligo, pathway analysis was carried out by MetaboAnalyst 5.0. We evaluated altered fatty acids within a pathway and its function of the pathway through variations in pivotal junction points of the pathway.

\section{RESULTS}

The anthropometric and clinical features of study subjects were presented in Supplementary Table S2. Samples for targeted metabolomics analysis were donated by a total of 48 vitiligo patients (24 males and 24 females, median and IQR age (34.5, 25.75-43.75) years) and 28 healthy controls ( 11 males and 17 females, median and IQR age (35, 26.5-40.75) years). The average VASI of patients with vitiligo was 1.76 . There were no significant differences in gender, age, body mass index (BMI), total cholesterol (TC), triglyceride (TG), high density lipoprotein cholesterol (HDL-C), or low density lipoprotein cholesterol (LDL-C), between the vitiligo group and healthy control group.

\section{Fatty Acid Metabolic Profiles of Vitiligo Patients Differed From That of Healthy Controls}

To clarify the connection between fatty acid metabolism and vitiligo, targeted metabolomics based on ultra-performance liquid chromatography-tandem mass spectrometry (UPLC-MS/MS) was applied on serum samples from 24 active vitiligo patients, 24 stable vitiligo patients and 28 healthy controls. Serological concentrations of 19 fatty acids of the cohort were shown in Supplementary Figure S1. Multivariate statistical analysis was carried out on the fatty acid 
metabolic profiles to demonstrate whether there were characteristic alterations that distinguished vitiligo patients from healthy controls. According to partial least squares discriminant analysis (PLS-DA), although principal components $1-5$ elucidated $66.5 \%$ of the variance of the LC-MS/MS data, there was no clear clustering that could distinguish vitiligo patients from healthy controls (Figure 1A). Moreover, the low R2 and Q2 values $(R 2=0.36$, $\mathrm{Q} 2=0.06$ ), which both indicated the quality of the PLS-DA model, suggested that the model was overfitted. Sequentially, to improve the diagnostic accuracy based on the fatty acid metabolic profiles, random forest (RF) algorithm was performed to identify similar regions that distinguished vitiligo patients and healthy individuals with an out-of-bag error of 0.276 for categorization (Figure 1B). RF analysis generated a series of fatty acids ranged by their importance to the classification program, and the top 15 fatty acids ascertained were shown in Figure 1C. Metabolite, especially ARA, was recognized as contributing to the division of groups. To summarize, the fatty acids that explain the difference of metabolic profiles between vitiligo patients and their healthy counterparts were determined.

To further clarify which specific fatty acid altered the metabolic profile of vitiligo patients, univariate statistical analysis was performed to hunt for differentially expressed fatty acids. As a result, serological levels of ALA were found to be the most significantly elevated in vitiligo patients compared with healthy controls, and levels of ARA, AA and behenic acid (BA) tended to diminish in the vitiligo group (Figure 2 and Supplementary Table S3). Additionally, binary logistic regression presented in Supplementary Table $\mathbf{S 4}$ revealed that ARA and AA were appreciated as a protective factor, while ALA was evaluated as a risk factor. These findings further specified the exact fatty acids that might be closely associated with vitiligo.

Furthermore, we asked whether some fatty acids could distinguish active vitiligo from stable vitiligo. Both the PLS-DA model and RF algorithm showed no clear differences among all fatty acids measured between progressive vitiligo and stable vitiligo (Supplementary Figures S2A, B). This disclosed that there were no marked differences in fatty acids among subgroups.

\section{ALA Is Positively Correlated With VASI}

Factors like sex, age, BMI, TG could influence fatty acids concentration in body fluids, thus the association between fatty acids and clinical characteristics should be considered.
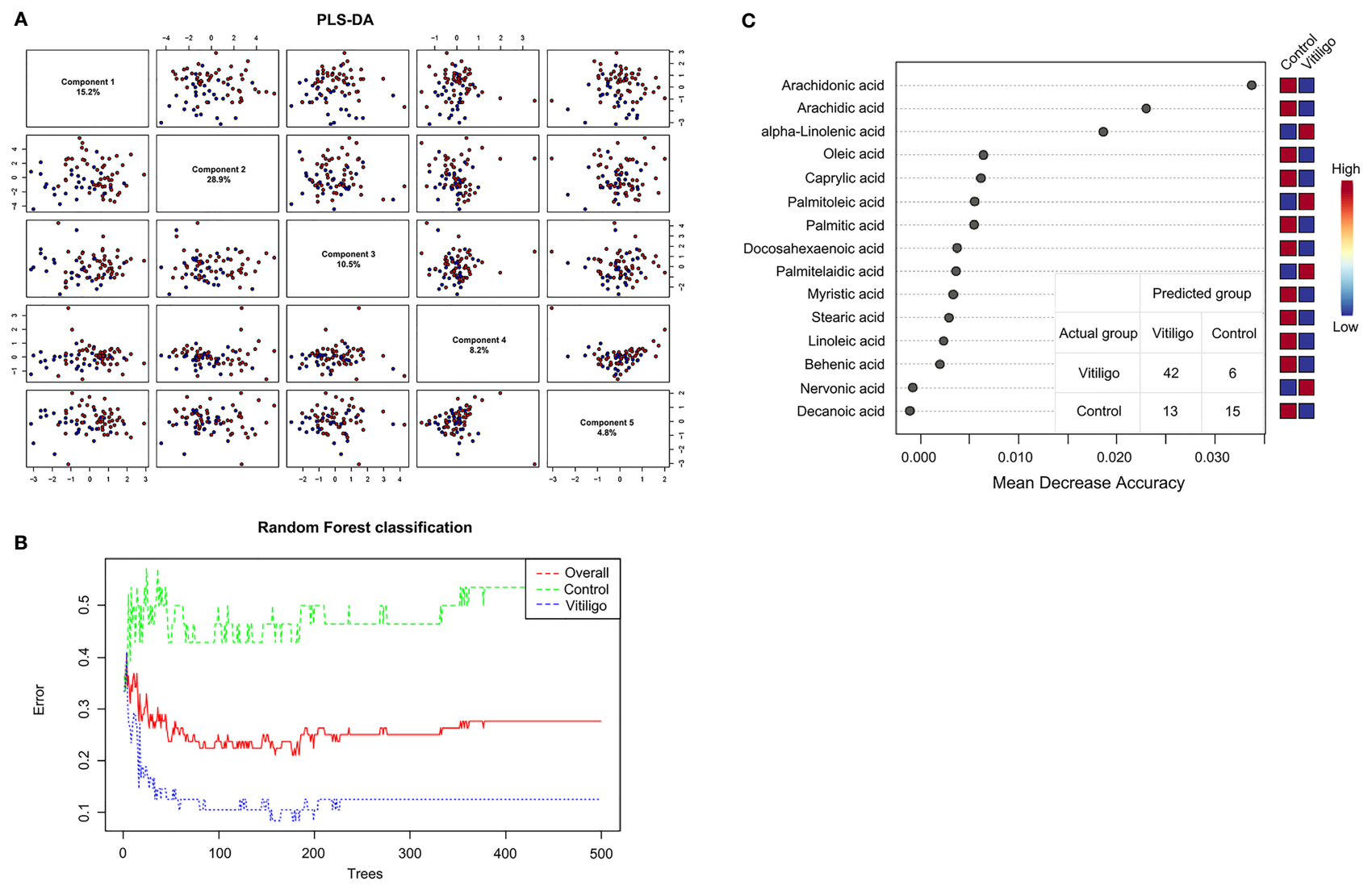

FIGURE 1 | Utilization of fatty acid profiles for classification of vitiligo patients and healthy controls by PLS-DA and RF analysis. (A) PLS-DA model shown the five principal components with the relative variances in pairwise scores plots. (B) Cumulative error rates by RF Classification. The red line represents the overall error rate; the vitiligo error rate and control error rate is shown as the blue line and green line, respectively. (C) Significant features confirmed by RF. The features are ranked by the mean decrease in classification accuracy when they are permuted. PLS-DA, partial least squares discriminant analysis; RF, random forest. 

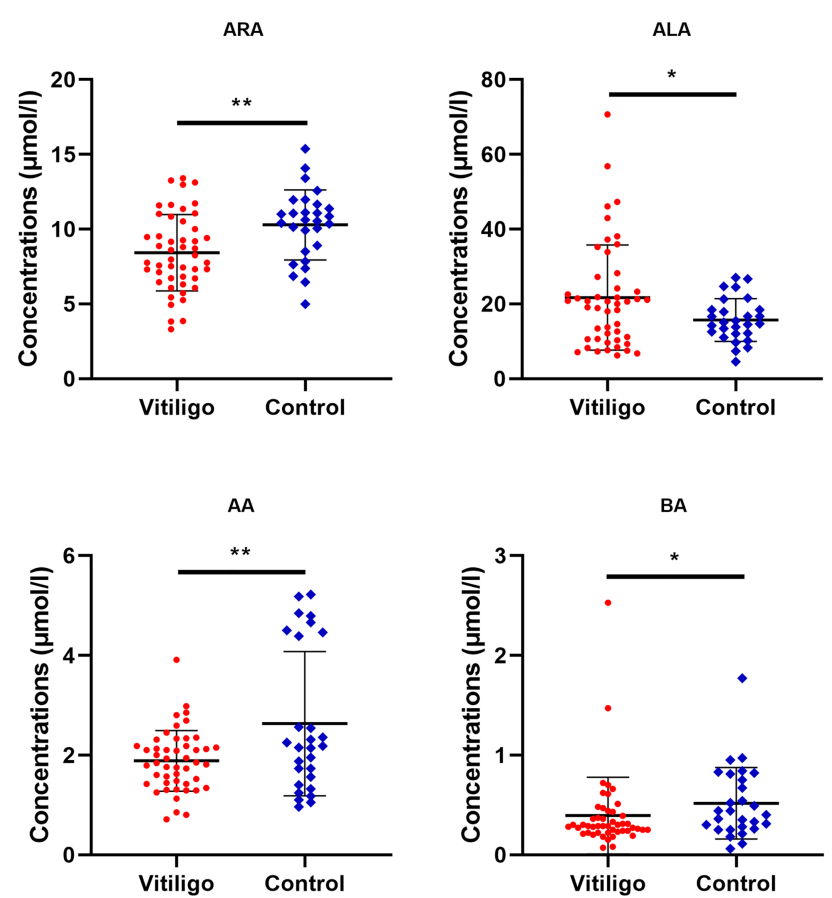

FIGURE 2 | Differential fatty acid levels in vitiligo patients as compared to healthy controls. The levels of ARA, ALA, AA and BA in vitiligo patients or healthy individuals were compared. The mean and standard deviation were represented by middle black line, the lower and upper black lines, respectively. ARA, arachidonic acid; ALA, alpha-linolenic acid; AA, arachidic acid; BA, behenic acid. ${ }^{\star} P<0.05,{ }^{\star \star} P<0.01$.

Even though strong correlation coefficients were found between long-chain saturated fatty acid (LC-SFA) and other fatty acids, notably, the strong correlation coefficient (Pearson correlation analysis, $\mathrm{r}=0.523, P<0.001)$ was discovered between VASI and ALA presented in Figure 3. What's more, a stronger correlation coefficient was uncovered between VASI and ALA $(r=0.692$, $P<0.001)$. These results indicated that the concentrations of some fatty acids, such as ALA, might partly reflect the severity of vitiligo.

\section{ARA Is the Most Characteristic Biomarker and Arachidonic Acid Metabolism Is the Most Enriched Metabolic Pathway in Vitiligo}

The diagnostic efficacy of significantly altered fatty acids was assessed by MetaboAnalyst 5.0. As a result, the area under the curve (AUC) of ARA was 0.709 as shown by the receiver operating characteristic curve (ROC), the AUC of other differentially expressed fatty acids, such as BA, AA and ALA were $0.657,0.616$, and 0.600 , respectively (Figure 4A). To improve the diagnostic accuracy of vitiligo, a combined predictive model was set up using four differential fatty acids, in which the AUCs were 0.817 (95\% confidence interval (CI): $0.719-0.915)$ with a sensitivity of $78.6 \%$ and specificity of $72.9 \%$ (Figure 4B). In order to make the prediction model more reliable and avoid over-fitting issues, we adopted a machine learning model of a 10-fold cross-validation and supporting vector machine (SVM). The AUC value of the model remained 0.789 and 0.763 , respectively (Figure 4B). These data indicated that fatty acids metabolic profiles, especially ARA, could be utilized to differentiate vitiligo patients from healthy individuals. Metabolic pathways are also critical in characterizing the metabolic profile of a disease in addition to metabolites. According to KEGG metabolic database disturbed arachidonic acid metabolism, alpha-linolenic acid metabolism and linoleic acid metabolism were markedly enriched (Supplementary Figure S3). Moreover, the FDR $<0.05$ of arachidonic acid metabolism denoted that it plays a critical role in patients with vitiligo.

\section{ARA Inhibits the Proliferation and Activation of CD8 ${ }^{+} \mathbf{T}$ Cells In Vitro}

Since ARA was significantly altered in vitiligo and served as a protective agent, we speculated that ARA supplementations might ameliorate the autoimmunity in vitiligo. Therefore, we focused on the influence of ARA on $\mathrm{CD}^{+} \mathrm{T}$ cells in patients with vitiligo in vitro. Firstly, the cell cytotoxicity assay indicated that there were no significant alterations in cell viability when the concentrations of ARA were under $50 \mu \mathrm{M}$ (Figure 5A). Thus, we chose $50 \mu \mathrm{M}$ as a limit concentration of ARA in our experiments in vitro.

Our result showed that ARA inhibited human $\mathrm{CD}^{+} \mathrm{T}$ cells proliferation stimulated by $\mathrm{CD} 3 / \mathrm{CD} 28$ monoclonal magnetic beads in a concentration-dependent manner, and the 


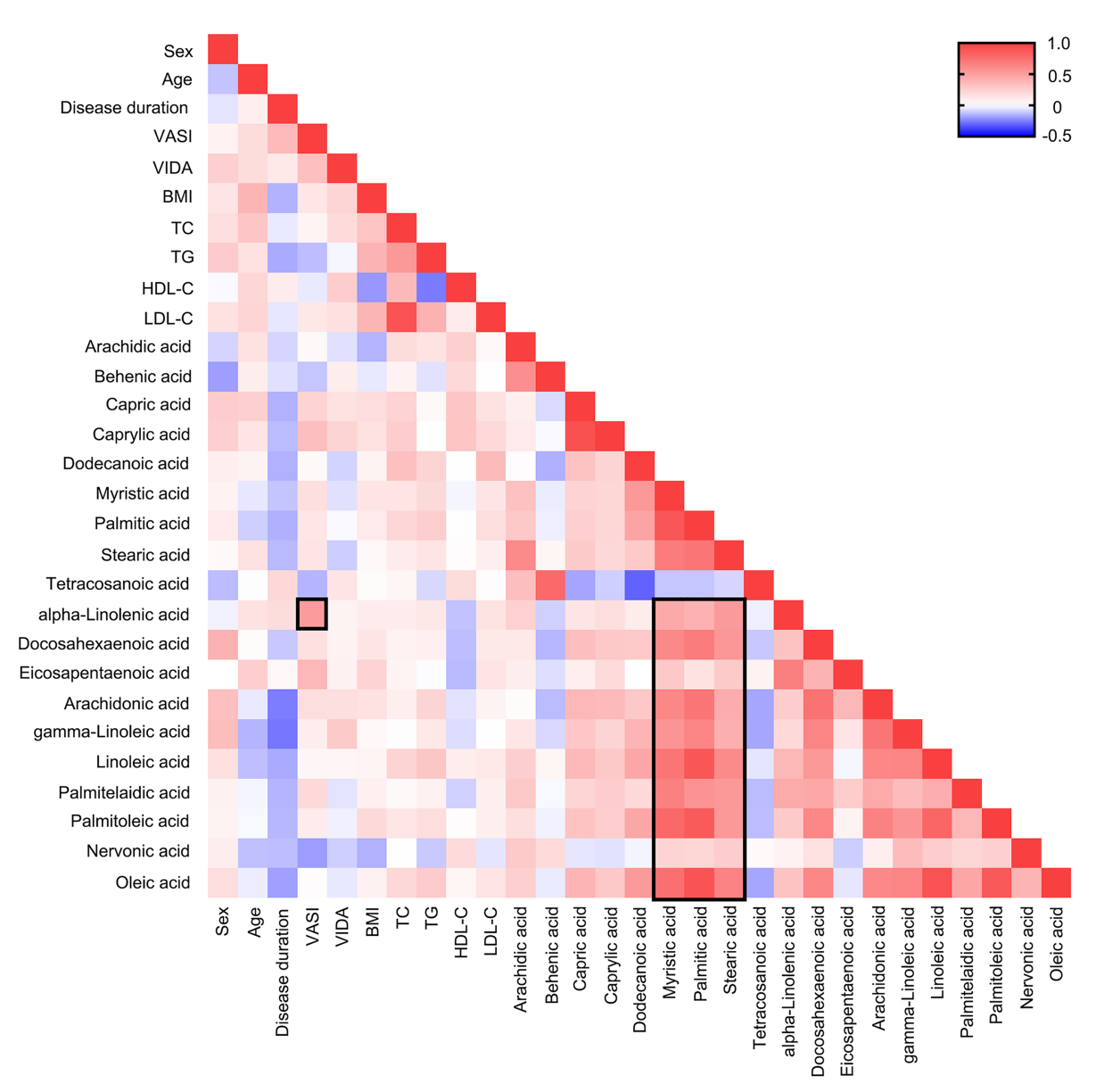

FIGURE 3 | Heat map of Pearson correlation analysis among fatty acids and clinical characteristics. Positive correlations are shown in red and negative correlations are shown in blue. The darker the color, the more relevant.

proliferation of $\mathrm{CD}^{+} \mathrm{T}$ cells was almost completely suppressed at $50 \mu \mathrm{M}$ (Figure 5B). Further, the expression of CD69 on $\mathrm{CD}^{+} \mathrm{T}$ cells was concentration-dependently inhibited at concentrations higher than $20 \mu \mathrm{M}$ (Figure 5C). These results indicated that ARA could inhibit the activation and proliferation of $\mathrm{CD}^{+} \mathrm{T}$ in a concentration-dependent manner.

\section{ARA and 5-LOX Inhibitor Suppress the Expression of Effector Molecules of CTLs}

We continued to clarify the effect of ARA on the expression of CD8 ${ }^{+}$ $\mathrm{T}$ effector molecules. Results disclosed that ARA significantly suppressed the expression of IFN- $\gamma$, granzyme B and perforin (Figures 6A-C). Thus, ARA might inhibit the effector function of $\mathrm{CD}^{+} \mathrm{T}$ cells.

Considering that cyclooxygenase (COX) pathway and lipoxygenase (LOX) pathway both participate in the arachidonic acid metabolism, we further employed celecoxib, COX-2 inhibitor, and NDGA, 5-LOX inhibitor, to specify the signaling pathway that contributes to ARA-mediated functional suppress of $\mathrm{CD} 8^{+} \mathrm{T}$ cells.
Our results showed that NDGA, instead of celecoxib, could inhibit the expression of IFN- $\gamma$, granzyme B and perforin (Figures 6A-C and Supplementary Figures S4A-C). Furthermore, we found the synergistic administration of ARA and NDGA performed better than their separate use in inhibiting the expression of IFN- $\gamma$, granzyme $\mathrm{B}$ and perforin in $\mathrm{CD}^{+}{ }^{+} \mathrm{T}$ cells activated by $\mathrm{CD} 3 / \mathrm{CD} 28$ monoclonal magnetic beads. Collectively, these results indicated that ARA and 5-LOX inhibitor might inhibit the expression of effector molecules in $\mathrm{CD}^{+} \mathrm{T}$ cells in vitro.

\section{DISCUSSION}

In the present study, we found a significant increase of ALA and decrease of ARA in vitiligo patients, and that supplementation with ARA or NDGA might suppress the self-reactive $\mathrm{CD}^{+} \mathrm{T}$ cells, characterized by the inhibition of the proliferation and activation of $\mathrm{CD}^{+} \mathrm{T}$ cells and lower expression levels of IFN- $\gamma$, granzyme $\mathrm{B}$ and perforin. 
A

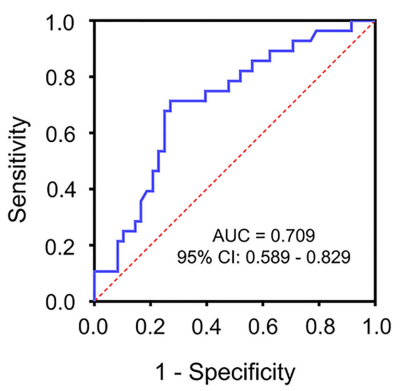

BA

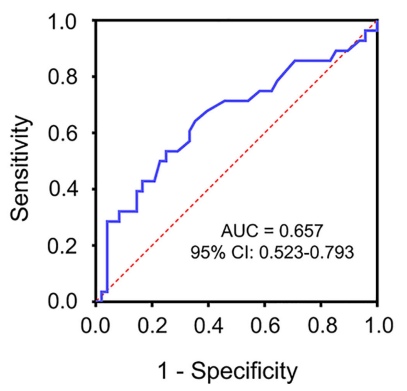

AA

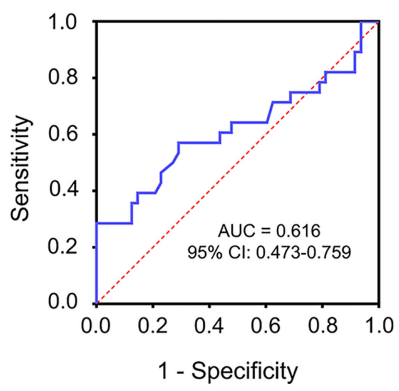

ALA

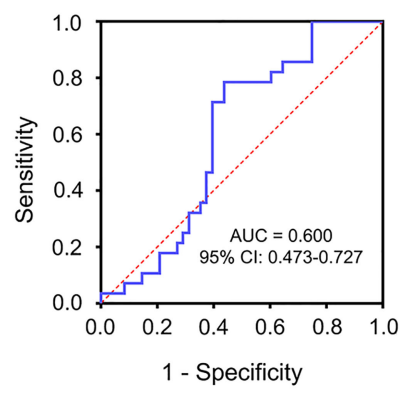

LR

B

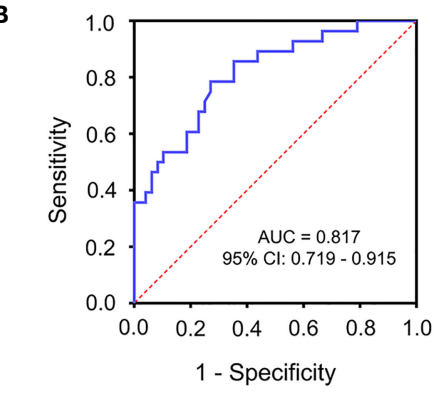

10-fold CV

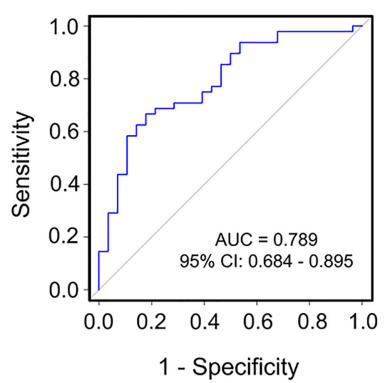

SVM

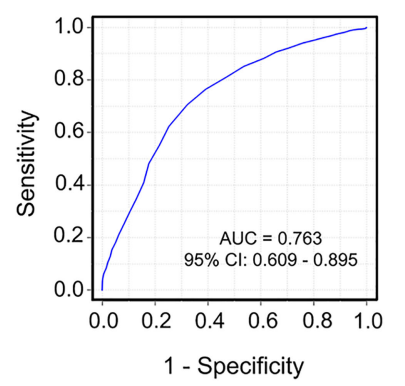

FIGURE 4 | Receiver operating characteristics curve for significantly altered serum metabolites in the comparison between vitiligo patients and healthy individuals. (A) ROC curves of ARA with $A \cup C=0.709$, BA with $A \cup C=0.657$, AA with $A \cup C=0.616$, ALA with AUC $=0.600$. (B) ROC curves of combined models selected from four differential fatty acids using LR (AUC =0.817), 10-fold CV (AUC =0.789), SVM (AUC =0.763), respectively. ROC, receiver operating characteristics; AUC, area under the curve; LR, logistic regression; 10-fold CV, 10-fold cross validation; SVM, supporting vector machine; Cl, confidence interval.

Circulating free fatty acids like ALA, EPA and ARA, are reportedly related to several autoimmune diseases. We evaluated the concentration of free fatty acids and observed increased levels of ALA, decreased levels of AA and BA in the serum of patients with vitiligo $(P<0.05)$. Unlike our results, Siro et al. discovered that the serum level of ALA was significantly reduced in patients with active vitiligo, while the expression of $\mathrm{AA}$ and $\mathrm{BA}$ were not markedly changed (23). The contradictory results might be attributed to either limited sample size or distinct measured methods in different studies, and the targeted metabolomics methods used in our study were more accurate than nontargeted data even though correction for signal drift (24).

Besides, the downregulated concentration of serum ARA in vitiligo was observed in our study, which paralleled previous reports (23). The main sources of ARA in the human body are synthesis from linoleic acid by phospholipase (PLA2, PLC and PLD) and supplement by dietary intake, while the disposition was metabolized to ARA-derived lipid mediators, such as prostaglandins (PGs), leukotrienes and epoxyeicosatrienoic acids (EETs). The reason for ARA decrease is still unclear. We surmise that metabolic exhaustion might provide an explanation considering the reportedly elevated levels of PGs (PGE2, PGD2 and PGF2 $\alpha$ ) in vitiligo lesions and leukotrienes in urine $(11,13)$, which are both metabolites of ARA. It's worth noting that abnormality of phospholipase in patients with vitiligo has not been previously reported, which needs further study.
In our present study, we also explored the relationship between the concentration of serum fatty acids and the vitiligo severity and activity. We observed that ALA was positively correlated with the disease severity (VASI scores), suggesting the potential of serum ALA as a new biomarker to assess vitiligo severity. Further pathway analysis disclosed that alpha-linolenic acid metabolism was critical to vitiligo pathogenesis. Previous global metabolomics profiles revealed that alpha-linolenic acid metabolism was significantly up-regulated in RA, and they suggest that long-term inflammation and nonsteroidal anti-inflammatory drugs intake may be responsible for the disturbed alpha-linolenic acid metabolism (25). Our study further validated that alpha-linolenic acid metabolic disturbance might be associated with chronic inflammatory status in vitiligo. Physiologically, alpha-linolenic acid metabolic pathway could be transformed into bioactivate mediators such as EPA and DHA. Thus, the observed positive correlation between ALA and VASI might corroborate with previous reports that EPA was inversely associated with the severity of RA and experimental autoimmune encephalomyelitis on account of the speculated low biotransformation rate of ALA in vitiligo $(26,27)$. Besides, several clinical experiments have disclosed that supplementation with EPA, DHA and their metabolites, resolvins, could contribute to the recovery of autoimmune diseases, such as diabetes and multiple sclerosis (28-30), which indicated that the role of metabolites of ALA in vitiligo still needs further exploration. Our study also revealed that there were no differences in free fatty acid between active vitiligo and stable 


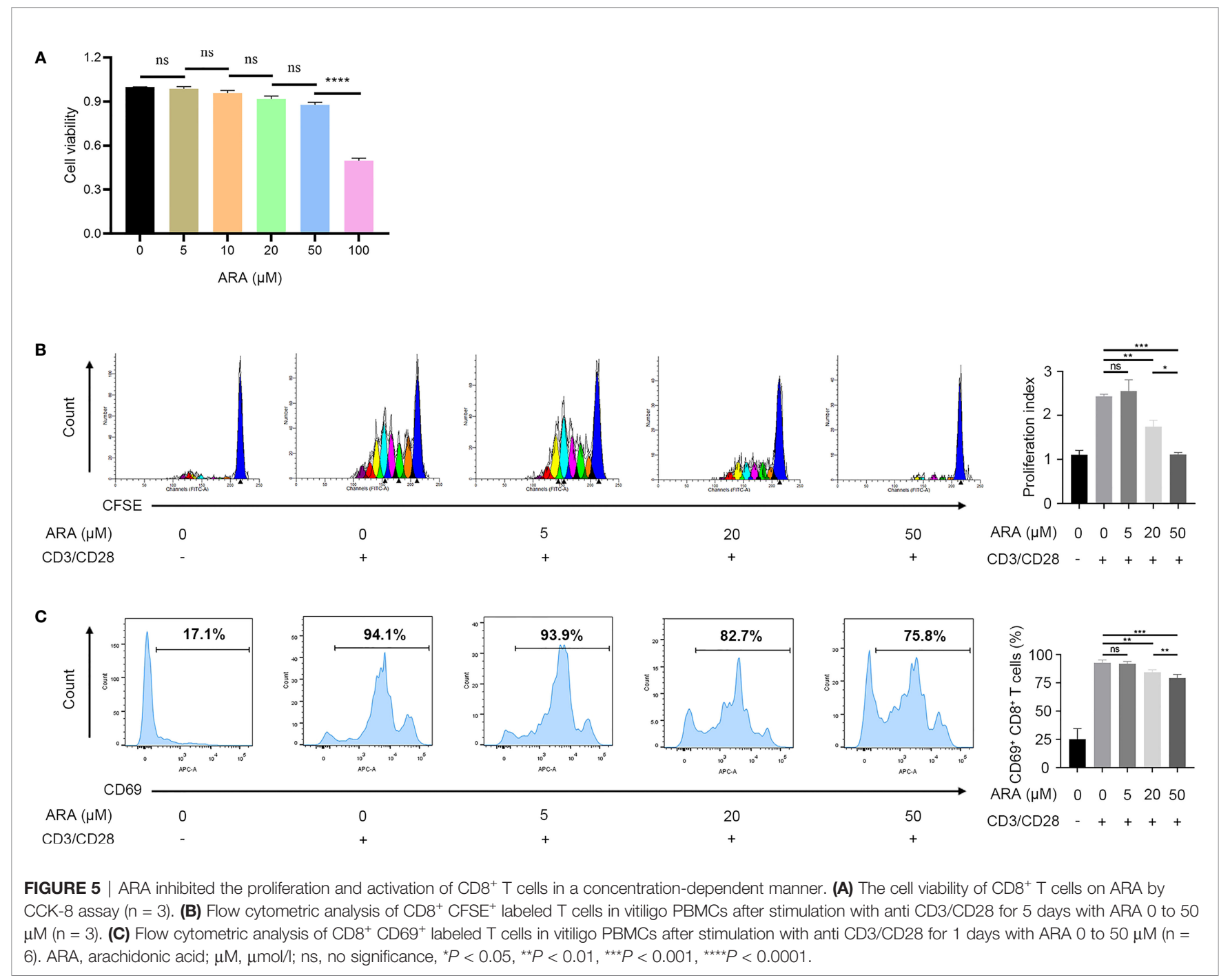

vitiligo. The association between fatty acid levels and vitiligo activity still needs further investigation owning to relatively small sample sizes in our research.

Our study also found that ARA was a sensitive biomarker for the predictive model of vitiligo. Although the diagnosis of vitiligo was relatively definitive by clinical observation and Wood's lamp, ARA, along with other unsaturated fatty acids could help reflect inflammation and autoimmunity status $(31,32)$. Previous studies confirmed that ARA was strongly associated with the risk of chronic inflammatory diseases and diabetes-associated autoimmunity (33-35). Our study revealed the role of ARA in the pathogenesis and treatment of vitiligo.

Vitiligo is an autoimmune skin disorder mainly mediated by autoreactive $\mathrm{CD}^{+} \mathrm{T}$ cells that lead to skin depigmentation. Our results indicated that ARA and its metabolic pathway were closely associated with autoimmune diseases. A preceding study reported that ARA, the terminal metabolite of linoleic acid, could suppress $\mathrm{CD} 8^{+} \mathrm{T}$ cell proliferation and activation in healthy individuals (36), which backed up our findings. Additionally, several pieces of research showed that ARA could inhibit the production of IFN- $\gamma$ in various diseases, such as metal-induced allergy diseases or neuroinflammatory disorders $(37,38)$. In our present study, we firstly demonstrated that ARA could inhibit the secretion of granzyme B and perforin.

Cyclooxygenase (COX) and lipoxygenase (LOX) metabolic pathway are regarded as the most important pathways in regulating arachidonic acid metabolism. Besides, metabolites of COX-2 and 5-LOX pathway have definitive immunomodulatory effects. A recent study found that celecoxib was effective in relieving swelling and inflammation in accordance with inhibition of IFN- $\gamma$ production in metal-induced allergy diseases (39). However, our results showed that celecoxib couldn't influence the expression of IFN- $\gamma$, as well as granzyme B and perforin generated by $\mathrm{CD}^{+} \mathrm{T}$ cells in vitiligo patients. It may partly result from PGs, the metabolites of COX-2 pathway, because low concentrations of PGE2 potentiate Th1 cells differentiation, while high concentrations work right reversely (40). Whereas NDGA, as a well-known 5-LOX inhibitor, could significantly inhibited the expression of IFN- $\gamma$, granzyme $\mathrm{B}$ and perforin of $\mathrm{CD}^{+} \mathrm{T}$ cells derived from vitiligo patients. In line with our findings, NDGA was 


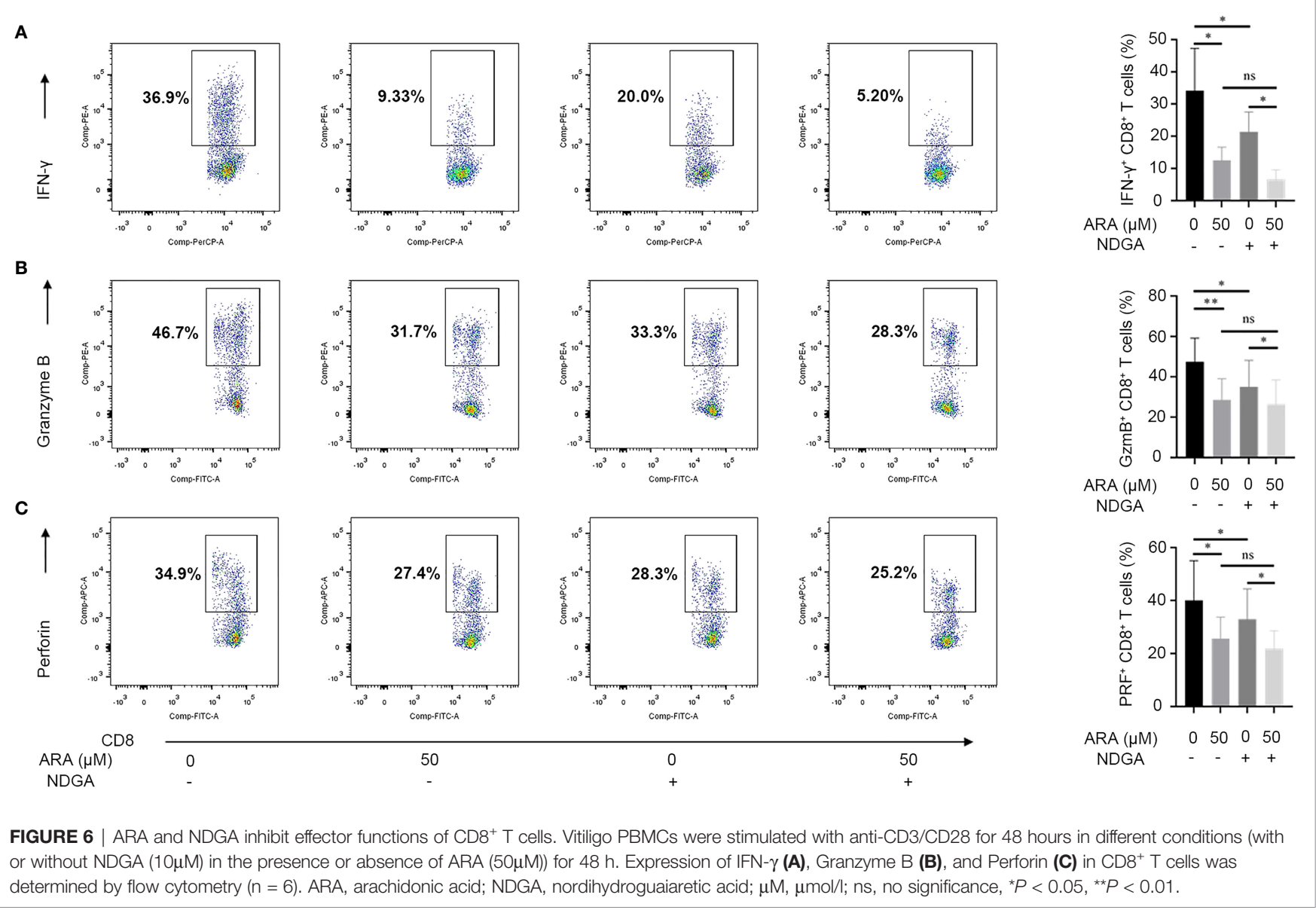

previously reported to inhibit IFN- $\gamma$-induced inflammatory response in vitro (41). In addition, their results suggest that NDGA regulates IFN- $\gamma$-mediated inflammation through mechanisms uncorrelated to the inhibition of 5-LOX metabolites. Inhibition of 5-LOX pathway could increase PGE2 generation through upregulating COX-2 pathway (42). And higher concentrations of PGE2 could inhibit the secretion of IFN- $\gamma$ to play a protective role in vitiligo. Therefore, inhibition of 5-LOX pathway in vitiligo treatment might be therapeutically exploitable.

Despite these findings, our study has several limitations. Firstly, although metabolomics can provide a comprehensive understanding of the disease, it still has some variability and inconsistencies due to the limited sample size (43). And a large number of population studies should be carried out in the future to avoid the limited statistical power. Secondly, our findings were derived from in vitro experiments, hence the effect of ARA and 5LOX inhibitor on $\mathrm{CD}^{+} \mathrm{T}$ cells and their capability in promoting repigmentation in vitiligo treatment still need further validation in vivo.

In summary, we performed a targeted metabolomics study and provided evidence that metabolic abnormality of fatty acids, especially ALA and ARA, were tightly associated with vitiligo. Moreover, ALA and ARA might be used for assessing vitiligo severity and predicting disease. Finally, our results found that
ARA and NDGA could inhibit the function of $\mathrm{CD}^{+} \mathrm{T}$ cells, which might offer a novel strategy for the treatment of vitiligo.

\section{DATA AVAILABILITY STATEMENT}

The original contributions presented in the study are included in the article/Supplementary Material. Further inquiries can be directed to the corresponding authors.

\section{ETHICS STATEMENT}

The studies involving human participants were reviewed and approved by Ethics Committee of Xijing Hospital. The patients/ participants provided their written informed consent to participate in this study.

\section{AUTHOR CONTRIBUTIONS}

ZY, JC, PD, CL, and SL came up with the design of the experiment. All authors contributed to the performance of 
the assays and acquisition of the sequencing and experimental data. ZY, JC, PD, and SL analyzed the data. ZY, JC, PD, CL, and SL wrote and revised the manuscript with input from all the authors.

\section{FUNDING}

This work was supported by the National Natural Science Foundation of China (No. 81930087, No. 12126606).

\section{REFERENCES}

1. Chen J, Li S, Li C. Mechanisms of Melanocyte Death in Vitiligo. Med Res Rev (2021) 41:1138-66. doi: 10.1002/med.21754

2. Taneja K, Taneja J, Kaur C, Patel S, Haldar D. Lipid Risk Factors in Vitiligo: Homocysteine the Connecting Link? Clin Lab (2020) 66(10). doi: 10.7754/ Clin.Lab.2020.200120

3. Kammeyer A, Willemsen KJ, Ouwerkerk W, Bakker WJ, Ratsma D, Pronk $\mathrm{SD}$, et al. Mechanism of Action of 4-Substituted Phenols to Induce Vitiligo and Antimelanoma Immunity. Pigment Cell Melanoma Res (2019) 32:540-52. doi: $10.1111 / \mathrm{pcmr} .12774$

4. Li S, Zhu G, Yang Y, Jian Z, Guo S, Dai W, et al. Oxidative Stress Drives CD8(+) T-Cell Skin Trafficking in Patients With Vitiligo Through CXCL16 Upregulation by Activating the Unfolded Protein Response in Keratinocytes. J Allergy Clin Immunol (2017) 140:177-89. doi: 10.1016/j.jaci.2016.10.013

5. Sahoo A, Lee B, Boniface K, Seneschal J, Sahoo SK, Seki T, et al. MicroRNA211 Regulates Oxidative Phosphorylation and Energy Metabolism in Human Vitiligo. J Invest Dermatol (2017) 137:1965-74. doi: 10.1016/j.jid.2017.04.025

6. Herbert D, Franz S, Popkova Y, Anderegg U, Schiller J, Schwede K, et al. High-Fat Diet Exacerbates Early Psoriatic Skin Inflammation Independent of Obesity: Saturated Fatty Acids as Key Players. J Invest Dermatol (2018) 138:1999-2009. doi: 10.1016/j.jid.2018.03.1522

7. Rodriguez-Carrio J, Lopez P, Sanchez B, Gonzalez S, Gueimonde M, Margolles A, et al. Intestinal Dysbiosis Is Associated With Altered ShortChain Fatty Acids and Serum-Free Fatty Acids in Systemic Lupus Erythematosus. Front Immunol (2017) 8:23. doi: 10.3389/fimmu.2017.00023

8. Ye Z, Shen Y, Jin K, Qiu J, Hu B, Jadhav RR, et al. Arachidonic AcidRegulated Calcium Signaling in T Cells From Patients With Rheumatoid Arthritis Promotes Synovial Inflammation. Nat Commun (2021) 12:907. doi: 10.1038/s41467-021-21242-Z

9. Tanacan E, Atakan N. Higher Incidence of Metabolic Syndrome Components in Vitiligo Patients: A Prospective Cross-Sectional Study. Bras Dermatol (2020) 95:165-72. doi: 10.1016/j.abd.2019.07.006

10. Marzabani R, Rezadoost H, Choopanian P, Kolahdooz S, Mozafari N, Mirzaie M, et al. Metabolomic Signature of Amino Acids in Plasma of Patients With non-Segmental Vitiligo. Metabolomics (2021) 17(10):92. doi: 10.1007/s11306021-01843-x

11. Liu W, Liu XY, Qian YT, Zhou DD, Liu JW, Chen T, et al. Urinary Metabolomic Investigations in Vitiligo Patients. Sci Rep (2020) 10:17989. doi: 10.1038/s41598-020-75135-0

12. Liang L, Li Y, Tian X, Zhou J, Zhong L. Comprehensive Lipidomic, Metabolomic and Proteomic Profiling Reveals the Role of Immune System in Vitiligo. Clin Exp Dermatol (2019) 44:e216-23. doi: 10.1111/ced.13961

13. Hensby CN, Shroot B, Schaefer H, Juhlin L, Ortonne JP. Prostaglandins in Human Skin Disease. Br J Dermatol (1983) 109(Suppl 25):22-5. doi: 10.1111/ j.1365-2133.1983.tb06813.x

14. Beyer K, Lie SA, Kjellevold M, Dahl L, Brun JG, Bolstad AI. Marine Omega-3, Vitamin D Levels, Disease Outcome and Periodontal Status in Rheumatoid Arthritis Outpatients. Nutrition (2018) 55-56:116-24. doi: 10.1016/ j.nut.2018.03.054

15. Veselinovic M, Vasiljevic D, Vucic V, Arsic A, Petrovic S, Tomic-Lucic A, et al. Clinical Benefits of N-3 PUFA and -Linolenic Acid in Patients With Rheumatoid Arthritis. Nutrients (2017) 9(4):325. doi: 10.3390/nu9040325

\section{ACKNOWLEDGMENTS}

We acknowledge the vitiligo patients and healthy controls for their blood samples and participation in this study.

\section{SUPPLEMENTARY MATERIAL}

The Supplementary Material for this article can be found online at: https://www.frontiersin.org/articles/10.3389/fimmu.2022.839167/ full\#supplementary-material

16. Arriens C, Hynan LS, Lerman RH, Karp DR, Mohan C. Placebo-Controlled Randomized Clinical Trial of Fish Oil's Impact on Fatigue, Quality of Life, and Disease Activity in Systemic Lupus Erythematosus. Nutr J (2015) 14:82. doi: 10.1186/s12937-015-0068-2

17. Das UN. Arachidonic Acid in Health and Disease With Focus on Hypertension and Diabetes Mellitus: A Review. J Adv Res (2018) 11:43-55. doi: 10.1016/j.jare.2018.01.002

18. Kang KW, Kim S, Cho YB, Ryu SR, Seo YJ, Lee SM. Endogenous N-3 Polyunsaturated Fatty Acids Are Beneficial to Dampen CD8(+) T CellMediated Inflammatory Response Upon the Viral Infection in Mice. Int J Mol Sci (2019) 20(18):4510. doi: 10.3390/ijms20184510

19. MonkJM, Liddle DM, De Boer AA, Brown MJ, Power KA, Ma DW, et al. Fish-OilDerived N-3 PUFAs Reduce Inflammatory and Chemotactic AdipokineMediated Cross-Talk Between Co-Cultured Murine Splenic CD8+ T Cells and Adipocytes. J Nutr (2015) 145:829-38. doi: 10.3945/jn.114.205443

20. Ni Q, Ye Z, Wang Y, Chen J, Zhang W, Ma C, et al. Gut Microbial Dysbiosis and Plasma Metabolic Profile in Individuals With Vitiligo. Front Microbiol (2020) 11:592248. doi: 10.3389/fmicb.2020.592248

21. Han J, Gagnon S, Eckle T, Borchers CH. Metabolomic Analysis of Key Central Carbon Metabolism Carboxylic Acids as Their 3-Nitrophenylhydrazones by UPLC/ESI-MS. Electrophoresis (2013) 34:2891-900. doi: 10.1002/elps. 201200601

22. Li J, Sun B, Guo Y, Wang X, Wang Y, Zhang H, et al. A Metabolite Array Technology for Precision Medicine. Anal Chem (2021) 93(14):5709-17. doi: 10.1021/acs.analchem.0c04686

23. Passi S, Grandinetti M, Maggio F, Stancato A, De Luca C. Epidermal Oxidative Stress in Vitiligo. Pigment Cell Res (1998) 11:81-5. doi: 10.1111/j.16000749.1998.tb00714.x

24. Ribbenstedt A, Ziarrusta H, Benskin JP. Development, Characterization and Comparisons of Targeted and non-Targeted Metabolomics Methods. PloS One (2018) 13:e207082. doi: 10.1371/journal.pone.0207082

25. Carlson AK, Rawle RA, Wallace CW, Adams E, Greenwood MC, Bothner B, et al. Global Metabolomic Profiling of Human Synovial Fluid for Rheumatoid Arthritis Biomarkers. Clin Exp Rheumatol (2019) 37:393-99.

26. Morin C, Blier PU, Fortin S. Eicosapentaenoic Acid and Docosapentaenoic Acid Monoglycerides are More Potent Than Docosahexaenoic Acid Monoglyceride to Resolve Inflammation in a Rheumatoid Arthritis Model. Arthritis Res Ther (2015) 17:142. doi: 10.1186/s13075-015-0653-y

27. Salvati S, Di Biase A, Attorri L, Di Benedetto R, Sanchez M, Lorenzini L, et al. Ethyl-Eicosapentaenoic Acid Ameliorates the Clinical Course of Experimental Allergic Encephalomyelitis Induced in Dark Agouti Rats. J Nutr Biochem (2013) 24:1645-54. doi: 10.1016/j.jnutbio.2013.02.005

28. Kouchaki E, Afarini M, Abolhassani J, Mirhosseini N, Bahmani F, Masoud SA, et al. High-Dose Omega-3 Fatty Acid Plus Vitamin D3 Supplementation Affects Clinical Symptoms and Metabolic Status of Patients With Multiple Sclerosis: A Randomized Controlled Clinical Trial. J Nutr (2018) 148:1380-86. doi: 10.1093/ jn/nxy116

29. Cadario F, Savastio S, Ricotti R, Rizzo AM, Carrera D, Maiuri L, et al. Administration of Vitamin D and High Dose of Omega 3 to Sustain Remission of Type 1 Diabetes. Eur Rev Med Pharmacol Sci (2018) 22:512-5. doi: 10.26355/eurrev_201801_14203

30. Poisson LM, Suhail H, Singh J, Datta I, Denic A, Labuzek K, et al. Untargeted Plasma Metabolomics Identifies Endogenous Metabolite With Drug-Like 
Properties in Chronic Animal Model of Multiple Sclerosis. J Biol Chem (2015) 290:30697-712. doi: 10.1074/jbc.M115.679068

31. Kuda O, Rossmeisl M, Kopecky J. Omega-3 Fatty Acids and Adipose Tissue Biology. Mol Aspects Med (2018) 64:147-60. doi: 10.1016/j.mam.2018. 01.004

32. Schwarz A, Bruhs A, Schwarz T. The Short-Chain Fatty Acid Sodium Butyrate Functions as a Regulator of the Skin Immune System. J Invest Dermatol (2017) 137:855-64. doi: 10.1016/j.jid.2016.11.014

33. Sztolsztener K, Chabowski A, Harasim-Symbor E, Bielawiec P, Konstantynowicz-Nowicka K. Arachidonic Acid as an Early Indicator of Inflammation During Non-Alcoholic Fatty Liver Disease Development. Biomolecules (2020) 10(8):1133. doi: 10.3390/biom10081133

34. Niinisto S, Takkinen HM, Erlund I, Ahonen S, Toppari J, Ilonen J, et al. Fatty Acid Status in Infancy is Associated With the Risk of Type 1 DiabetesAssociated Autoimmunity. Diabetologia (2017) 60:1223-33. doi: 10.1007/ s00125-017-4280-9

35. Korotkova M, Lundberg IE. The Skeletal Muscle Arachidonic Acid Cascade in Health and Inflammatory Disease. Nat Rev Rheumatol (2014) 10:295-303. doi: 10.1038/nrrheum.2014.2

36. Alnajjar A, Chabane SD, Abuharfeil N, Hudaib M, Aburjai T. Effect of N-3 and N-6 Polyunsaturated Fatty Acids on Lymphocyte Proliferation, Interleukin Production and Phospholipid Fatty Acids Composition in Type 2 Diabetic and Healthy Subjects in Jordan People. Prostaglandins Leukot Essent Fatty Acids (2006) 74:347-56. doi: 10.1016/j.plefa.2006.03.001

37. Chiu YJ, Lin SA, Chen WL, Lin TH, Lin CH, Yao CF, et al. Pathomechanism Characterization and Potential Therapeutics Identification for SCA3 Targeting Neuroinflammation. Aging (Albany NY) (2020) 12:23619-46. doi: 10.18632/aging.103700

38. Chou JP, Ramirez CM, Ryba DM, Koduri MP, Effros RB. Prostaglandin E2 Promotes Features of Replicative Senescence in Chronically Activated Human CD8+ T Cells. PloS One (2014) 9:e99432. doi: 10.1371/journal.pone.0099432

39. Sitalaksmi RM, Ito K, Ogasawara K, Suto Y, Itabashi M, Ueda K, et al. COX-2 Induces $\mathrm{T}$ Cell Accumulation and IFN-Gamma Production During the
Development of Chromium Allergy. Autoimmunity (2019) 52:228-34. doi: $10.1080 / 08916934.2019 .1662404$

40. Sreeramkumar V, Fresno M, Cuesta N. Prostaglandin E2 and T Cells: Friends or Foes? Immunol Cell Biol (2012) 90:579-86. doi: 10.1038/icb.2011.75

41. Jeon SB, Ji KA, You HJ, Kim JH, Jou I, Joe EH. Nordihydroguaiaretic Acid Inhibits IFN-Gamma-Induced STAT Tyrosine Phosphorylation in Rat Brain Astrocytes. Biochem Biophys Res Commun (2005) 328:595-600. doi: 10.1016/ j.bbrc.2005.01.025

42. He C, Wu Y, Lai Y, Cai Z, Liu Y, Lai L. Dynamic Eicosanoid Responses Upon Different Inhibitor and Combination Treatments on the Arachidonic Acid Metabolic Network. Mol Biosyst (2012) 8:1585-94. doi: 10.1039/c2mb05503a

43. Kohler I, Hankemeier T, van der Graaf PH, Knibbe CAJ, van Hasselt JGC. Integrating Clinical Metabolomics-Based Biomarker Discovery and Clinical Pharmacology to Enable Precision Medicine. Eur J Pharm Sci (2017) 109S: S15-21. doi: 10.1016/j.ejps.2017.05.018

Conflict of Interest: The authors declare that the research was conducted in the absence of any commercial or financial relationships that could be construed as a potential conflict of interest.

Publisher's Note: All claims expressed in this article are solely those of the authors and do not necessarily represent those of their affiliated organizations, or those of the publisher, the editors and the reviewers. Any product that may be evaluated in this article, or claim that may be made by its manufacturer, is not guaranteed or endorsed by the publisher.

Copyright $\odot 2022 \mathrm{Ye}$, Chen, Du, Ni, Li, Zhang, Wang, Cui, Yi, Li and Li. This is an open-access article distributed under the terms of the Creative Commons Attribution License (CC BY). The use, distribution or reproduction in other forums is permitted, provided the original author(s) and the copyright owner(s) are credited and that the original publication in this journal is cited, in accordance with accepted academic practice. No use, distribution or reproduction is permitted which does not comply with these terms. 